\title{
Comparação de Marcadores para Estimativas de Produção Fecal e de Fluxo de Digesta em Bovinos 1
}

\section{Telma Teresinha Berchielli², Simone Gisele de Oliveira ${ }^{3}$, Elma Neide Vasconcelos Martins Carrilho $^{4}$, José Valmir Feitosa ${ }^{3}$, André Dias Lopes 5}

\begin{abstract}
RESUMO - Foram realizados três experimentos procurando avaliar a utilização de dois marcadores internos (FDN e FDA indigestíveis), obtidos por meio da incubação in vitro e in situ, e de um marcador externo (óxido crômico) para estimativa da produção fecal e do fluxo da digesta duodenal em bovinos. Para análise dos dados, adotou-se o delineamento em blocos inteiramente ao acaso, sendo os tratamentos distribuídos em esquema fatorial, constituindo cinco marcadores e três volumosos. Os teores de FDN e FDA indigestíveis mostraram-se variáveis para cada volumosos, independentemente da metodologia utilizada (in vitro ou in situ), indicando que possivelmente a incubação por 144 horas não reproduz a fração indigestível total. As estimativas de produção fecal e de fluxo da digesta duodenal, obtidas por intermédio dos marcadores avaliados, apresentaram comportamento bastante diferenciado de acordo com cada volumoso. Os marcadores internos (FDN e FDA indigestíveis) podem ser utilizados como preditores dos parâmetros avaliados, desde que alguns cuidados sejam tomados na sua determinação.
\end{abstract}

Palavras-chave: FDA indigestível, FDN indigestível, marcadores, método in situ, método in vitro, óxido crômico

\section{Comparison of Markers Used to Estimate Fecal Production and Digesta Flow of Cattle}

ABSTRACT - In order to estimate fecal production and digesta flow of cattle, three assays were carried out using indigestible NDF and ADF as internal markers, applying in vitro and in situ incubation procedures, as well as chromium oxide as an external marker. The experiment was a randomized block design in a factorial arrangement. Indigestible NDF and ADF contents exhibited high variation despite the methodology employed (in vitro or in situ). These results indicate that, eventually, 144-h incubation time does not reproduce the indigestible total fraction. The estimates of fecal production and duodenal digesta flow, calculated in function of the marker used, presented a variable behavior according to the roughage, which implies its effect on the marker concentration. Regarding important criteria involved in their determination, these internal markers can be used as predictors of the evaluated parameters.

Key Words: ADF indigestible, chromium oxide, in situ incubation, in vitro incubation, markers, NDF indigestible

\section{Introdução}

$\mathrm{Na}$ tentativa de estimar de maneira confiável as exigências dos animais e o valor nutritivo dos alimentos, sistemas de energia metabolizável (AFRC, 1993) e de proteína (AFRC, 1993, NRC, 2001) têm sido desenvolvidos. No entanto, esses sistemas se baseiam nos dados derivados de estudos fundamentados nas medidas do destino dos nutrientes digeridos no rúmen e na suplementação destes para o animal hospedeiro. Segundo Ahvenjärvi et al. (2003), os erros associados à predição do fornecimento dos nutrientes usando modelos são baseados em medidas de fluxo duodenal de nutrientes e, ao melhorar a precisão das medidas de fluxo, os erros de predição do suprimento de nutrientes para o animal hospedeiro serão reduzidos.
Uma variedade de fatores influencia os resultados de estudos realizados para medir o desaparecimento ruminal e o fluxo de nutrientes para o intestino, entre eles a ingestão de alimentos, o marcador utilizado, a coleta de amostra, o delineamento estatístico (Titgemeyer, 1997), as recuperações incompletas de marcadores, a variação na taxa de passagem do rúmen e as amostras não representativas (Merchen, 1988).

A produção fecal pode ser determinada a partir da coleta total ou da recuperação do marcador fecal, ao passo que as medidas de fluxo de digesta duodenal requerem cânula reentrante, dificultando os trabalhos. No entanto, estimativas de fluxo de digesta têm sido obtidas com a coleta de amostras de digesta via cânulas duodenais em $\mathrm{T}$ e uso de marcadores.

\footnotetext{
${ }^{1}$ Financiado pela FAPESP.

2 Professora do Departamento de Zootecnia - FCAV/UNESP - Jaboticabal, SP, Pesquisadora do CNPq (ttberchi@fcav.unesp.br).

3 Programa de Pós-graduação em Zootecnia, FCAV/UNESP - Jaboticabal, SP. Bolsista do CNPq.

${ }^{4}$ Bolsa PRODOC/CAPES, FCAV/UNESP - Jaboticabal, SP.

${ }^{5}$ Aluno do curso de graduação em Zootecnia, FCAV/UNESP - Jaboticabal, SP, Bolsista FAPESP.
} 
Vários marcadores vêm sendo empregados em estudos de metabolismos animal (Owens \& Hanson, 1992). Marcadores internos apresentam a vantagem de serem componentes indigestíveis presentes no alimento, não havendo, portanto, a necessidade de prévio preparo. Os componentes indigestíveis da parede celular, como fibra em detergente neutro (FDN) e fibra em detergente ácido (FDA) têm sido usados (Ítavo et al., 2002a; Zeoula et al., 2002), podendo ser obtidos pela incubação ruminal em sacos de náilon ou incubação in vitro em líquido ruminal (Freitas et al., 2002).

O óxido crômico, apesar de apresentar alguns problemas, como recuperação diferente de $100 \%$, variação na recuperação fecal entre animais e concentração nas fezes variável no decorrer do dia, é o marcador externo mais comumente empregado em estudos de digestão por ser de baixo custo, ser rapidamente incorporado à dieta e analisado com relativa facilidade (Titgemeyer, 1997).

Objetivou-se, com o presente estudo, avaliar a utilização de marcadores internos (FDN e FDA indigestível) obtidos pela incubação in vitro e in situ e do marcador externo (óxido crômico) para a estimativa da produção fecal e fluxo de digesta duodenal em bovinos.

\section{Material e Métodos}

As estimativas de fluxo de digesta duodenal e de produção fecal foram determinadas em três experimentos, havendo variação no tipo de volumoso empregado (silagem de milho, feno de Tifton-85 e canade-açúcar). Foram utilizados para cada experimento oito bovinos mestiços castrados, com peso médio de $340 \mathrm{~kg}$, providos de cânula no rúmen e duodeno proximal. Os experimentos foram divididos em quatro períodos de 21 dias (14 dias para adaptação dos animais às dietas e sete para coleta de amostras). Durante o período de adaptação, os animais foram mantidos em baias individuais semicobertas, com cochos e bebedouro, e, no período de coleta de dados, foram transferidos para gaiolas de estudos metabólicos, com piso ripado de madeira e providas de bandeja para coleta total de fezes.

A produção fecal diária foi obtida pela coleta total das fezes das bandejas, efetuada diariamente às $7 \mathrm{~h} 30$, durante sete dias. As fezes foram pesadas, homogeneizadas, coletando-se uma amostra representativa, para determinação da concentração dos marcadores.
Para determinação do fluxo diário de matéria seca no duodeno, coletaram-se, aproximadamente, $200 \mathrm{~mL}$ de digesta duodenal, via cânula, durante quatro dias em intervalos de 8 horas, para compor uma amostra representativa do fluxo diário do marcador.

O consumo de matéria seca e de marcadores foi determinado pelo controle diário do alimento consumido pelos animais. A quantidade fornecida foi controlada de forma a não permitir sobras no cocho e não possibilitar a seleção das dietas por parte dos animais. As amostras do alimento fornecido, das fezes e da digesta duodenal permaneceram armazenadas a $-10^{\circ} \mathrm{C}$ e, posteriormente, foram secas em estufa a $55^{\circ} \mathrm{C}$ com ventilação forçada, durante 72 horas, e moídas a $5 \mathrm{~mm}$, para posterior determinação da fração indigestível.

Estimativas de fluxo duodenal e de produção fecal foram obtidas com dois marcadores internos (FDN e FDA indigestíveis) e óxido crômico como marcador externo.

O óxido crômico foi fornecido em duas doses diárias $(5 \mathrm{~g})$ diretamente no rúmen, via cânula, iniciando-se cinco dias antes das coletas para que ocorresse o equilíbrio do marcador no trato gastrintestinal. A digestão das amostras de cana-de-açúcar, para posterior análise da concentração de cromo, foi obtida conforme Carrilho et al. (2003) e a determinação do cromo nas amostras de digesta duodenal e fezes, segundo Williams et al. (1962).

A FDN e FDA in vitro foram obtidas após a incubação dos alimentos, da digesta duodenal e da fezes por 144 horas em fluido ruminal, conforme Berchielli et al. (2000). A técnica do saco de náilon foi utilizada para determinação da FDN e FDA in situ utilizando bolsas de tecido de náilon com dimensões de 7 x $14 \mathrm{~cm}$ e diâmetro de poros de $50 \mathrm{~mm}$. Amostras de alimentos, de digesta duodenal e de fezes foram incubadas no rúmen de oito bovinos por 144 horas. Após a incubação ruminal, as bolsas foram lavadas e submetidas à secagem em estufa com ventilação forçada a $55^{\circ} \mathrm{C}$, por 72 horas.

As determinações de fibra em detergente neutro (FDN) e fibra em detergente ácido (FDA) foram realizadas de acordo com o método de Van Soest et al. (1991), utilizando-se o aparelho ANKON ${ }^{200}$ (Ankom Technology Corp., Fairport, NY, USA).

A produção fecal foi estimada baseando-se na razão entre a quantidade do marcador administrado ao animal e sua concentração nas fezes: 
Produção fecal $(\mathrm{g} /$ dia $)=\frac{\text { Gramas de marcador ingerido }}{\text { Concetração de marcador nas fezes }}$

O fluxo da digesta duodenal, baseado na concentração do marcador externo, foi calculado pela equação:

$\underset{\text { de MS }}{\text { Fluxo }}=\frac{\text { Gramas de marcador fornecido }}{\text { Concent. de marcador }(\mathrm{g}) / \mathrm{g} \text { de MS duodenal }}$

A estimativa do fluxo duodenal de matéria seca obtida por meio do marcador interno foi baseada na produção fecal e na relação entre a concentração do marcador nas fezes e no duodeno, segundo a equação:

$$
\begin{aligned}
& \text { Fluxo } \\
& \text { duodenal } \\
& \text { de MS }
\end{aligned}=\text { MSF } \times \frac{\% \text { FDNiv }(\text { FD Nis }) \text { ou FD Aiv (FD Ais }) \mathrm{F}}{\% \text { FDNiv (FD Nis)ou FD Aiv(FD Ais }) \text { DD }}
$$

em que: FDNiv - FDN obtida após incubação in vitro por 144 horas; FDNis - FDN obtida após incubação in situ por 144 horas; FDAiv - FDA obtida após incubação in vitro por 144 horas; FDAis - FDA obtida após incubação in situ por 144 horas; MSF - MS fecal obtida a partir da coleta total; F - fezes; DD - digesta duodenal.

Os coeficientes de digestibilidade total e ruminal foram obtidos pela diferença entre a quantidade de alimento ingerido e a produção fecal e fluxo de MS digesta duodenal, respectivamente:

$\left.\begin{array}{l}\text { Coeficiente de }=100 \times\left[\frac{\text { MS ingerida }- \text { MS fecal ou MS duodenal }}{\text { MS ingerida }}\right. \\ \text { digestão }\end{array}\right]$

Adotou-se o delineamento de blocos inteiramente casualizados, considerando-se o período como fator bloco. Os tratamentos foram distribuídos em esquema fatorial 5 x 3 (cinco marcadores e três volumosos) e as médias foram analisadas pelo teste Tukey a 5\% de significância pelo PROC GLM (SAS, 2003).

A análise de contraste foi realizada procurando confrontar os diferentes teores de marcadores internos (FDN x FDA) e as diferentes metodologias (in vitro $\mathrm{x}$ in situ) empregadas para averiguar possíveis efeitos dos métodos de obtenção do marcador sobre sua concentração no volumoso.

\section{Resultados e Discussão}

Um marcador ideal é aquele que prediz precisamente a digestibilidade no trato total e, em particular, fornece informações exatas sobre a extensão e direção dos efeitos induzidos pelas dietas sem modificar o sentido dos efeitos dos tratamentos. Administração do marcador é exposta a variações em razão da inconsistência na taxa de infusão ou, ocasionalmente, de vazamento através da cânula. Quando complexados com outros compostos, como o EDTA, relativamente menor quantidade de cromo pode ser adsorvida pelo trato gastrintestinal e subseqüentemente excretado na urina (Faichney, 1975; Siddons et al., 1985).

Houve efeito da metodologia para obtenção do marcador (in situ e in vitro), indicando que maior atenção deve ser dada ao considerar comparações entre estimativas obtidas pelas diferentes metodologias (Tabela 1).

Os teores de FDN e FDA indigestíveis foram diferentes dos observados na literatura (Berchielli et al., 2000; Zeoula et al., 2002). No entanto, é importante salientar que o tempo de incubação também tem variado muito entre trabalhos, sendo encontrados tempos de 72 e 144 horas (Berchielli et al., 2000), 96 horas (Ruiz et al., 2001), 192 horas (Zeoula et al., 2002) e 288 horas (Huhtanen et al., 1994), não havendo homogeneidade dos dados, o que leva a crer que os erros de metodologia de análise continuam a ser os maiores problemas. Lippke et al. (1986) relatam que boa parte da variabilidade dos resultados obtidos com marcadores internos indigestíveis pode ser atribuída à falta de padronização no método de determinação. Huhtanen et al. (1994) observaram que a incubação ruminal por 288 horas é o ideal, uma vez que os autores verificaram maiores teores de FDNiv e FDAiv quando incubados por 96 horas em relação a incubação por 288 horas.

A variação observada para os teores dos marcadores nos volumosos refletiu no consumo pelos animais (Tabela 2), afetando os parâmetros estudados, uma vez que as estimativas da produção fecal consideram a ingestão diária do marcador. A silagem de milho apresentou maior consumo de matéria seca em relação aos demais volumosos, resultando em maior consumo dos marcadores. 
Tabela 1 - Contraste entre as metodologias para obtenção dos marcadores internos Table 1 - Metodology contrast to determine internal markers

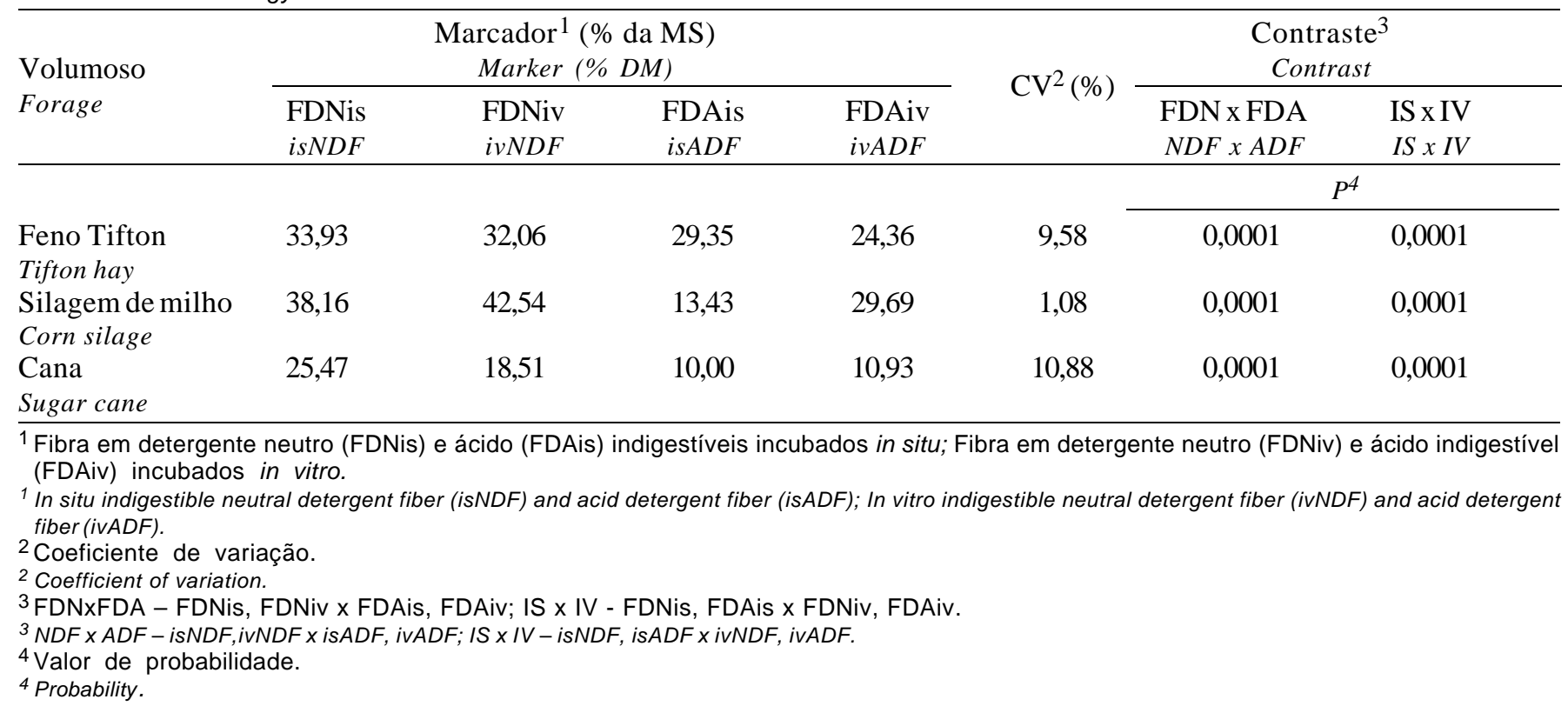

Tabela 2 - Consumo de matéria seca e marcadores $(\mathrm{kg} / \mathrm{d})$ para os volumosos avaliados

Table 2 - Forage dry matter and markers intake $(\mathrm{kg} / \mathrm{d})$

\begin{tabular}{|c|c|c|c|c|c|c|}
\hline \multirow{2}{*}{$\begin{array}{l}\text { Volumoso } \\
\text { Forage }\end{array}$} & \multirow{2}{*}{$\begin{array}{c}\mathrm{CMS}^{1} \\
D M I\end{array}$} & \multicolumn{4}{|c|}{$\begin{array}{c}\text { Marcador }^{2} \\
\text { Marker }\end{array}$} & \multirow{2}{*}{$\mathrm{CV}^{3}(\%)$} \\
\hline & & $\begin{array}{l}\text { FDNis } \\
\text { isNDF }\end{array}$ & $\begin{array}{l}\text { FDNiv } \\
i v N D F\end{array}$ & $\begin{array}{l}\text { FDAis } \\
\text { isADF }\end{array}$ & $\begin{array}{l}\text { FDAiv } \\
i v A D F\end{array}$ & \\
\hline $\begin{array}{l}\text { Feno tifton } \\
\text { Tifton hay }\end{array}$ & 4,76 & $1,61 \mathrm{a}$ & $1,52 \mathrm{a}$ & $1,38 \mathrm{~b}$ & $1,15 \mathrm{c}$ & 9,34 \\
\hline $\begin{array}{l}\text { Silagem de milho } \\
\text { Corn silage }\end{array}$ & 6,25 & $2,38 \mathrm{~b}$ & $2,66 \mathrm{a}$ & $0,84 \mathrm{~d}$ & $1,85 \mathrm{c}$ & 12,39 \\
\hline \multicolumn{7}{|c|}{$\begin{array}{l}{ }^{1} \text { Consumo de matéria seca. } \\
{ }^{1} \text { Dry matter intake. } \\
{ }^{2} \text { Fibra em detergente neutro (FDNis) e ácido (FDAis) indigestíveis incubados in situ; Fibra em detergente neutro (FDNiv) e ácido indigestível } \\
\text { (FDAiv) incubados in vitro. } \\
2 \text { In situ indigestible neutral detergent fiber (isNDF) and acid detergent fiber (isADF); In vitro indigestible neutral detergent fiber (ivNDF) and acid detergent } \\
\text { fiber (ivADF). }\end{array}$} \\
\hline
\end{tabular}

Quando utilizado o feno de Tifton 85 como volumoso, a produção fecal obtida pelos marcadores FDNiv e FDAis não diferiu da coleta total (CT) (Tabela 3). O FDAiv subestimou e o FDNis e o óxido crômico superestimaram a produção fecal. Para silagem de milho, apenas as estimativas por meio do FDAiv não diferiram da coleta total.

As estimativas de produção fecal utilizando marcadores internos diferiram da coleta total para a cana-de-açúcar, enquanto o marcador externo não apresentou diferença. Portanto, os marcadores apresentam comportamento diferenciado de acordo com cada volumoso estudado. Entre as razões para essa diferença, possivelmente a constituição da fibra de cada volumoso desempenha importante papel, podendo afetar sua taxa e extensão de degradação. Dessa forma, diferentes tempos de incubação podem ser necessários, dependendo de como é constituída a porção fibrosa de cada volumoso. 
Tabela 3 - Produção fecal $(\mathrm{kg} / \mathrm{d}$ ) obtida pela coleta total e estimada com uso de marcadores

Table 3 - Fecal production $(\mathrm{kg} / \mathrm{d})$ by total collection and estimate with markers

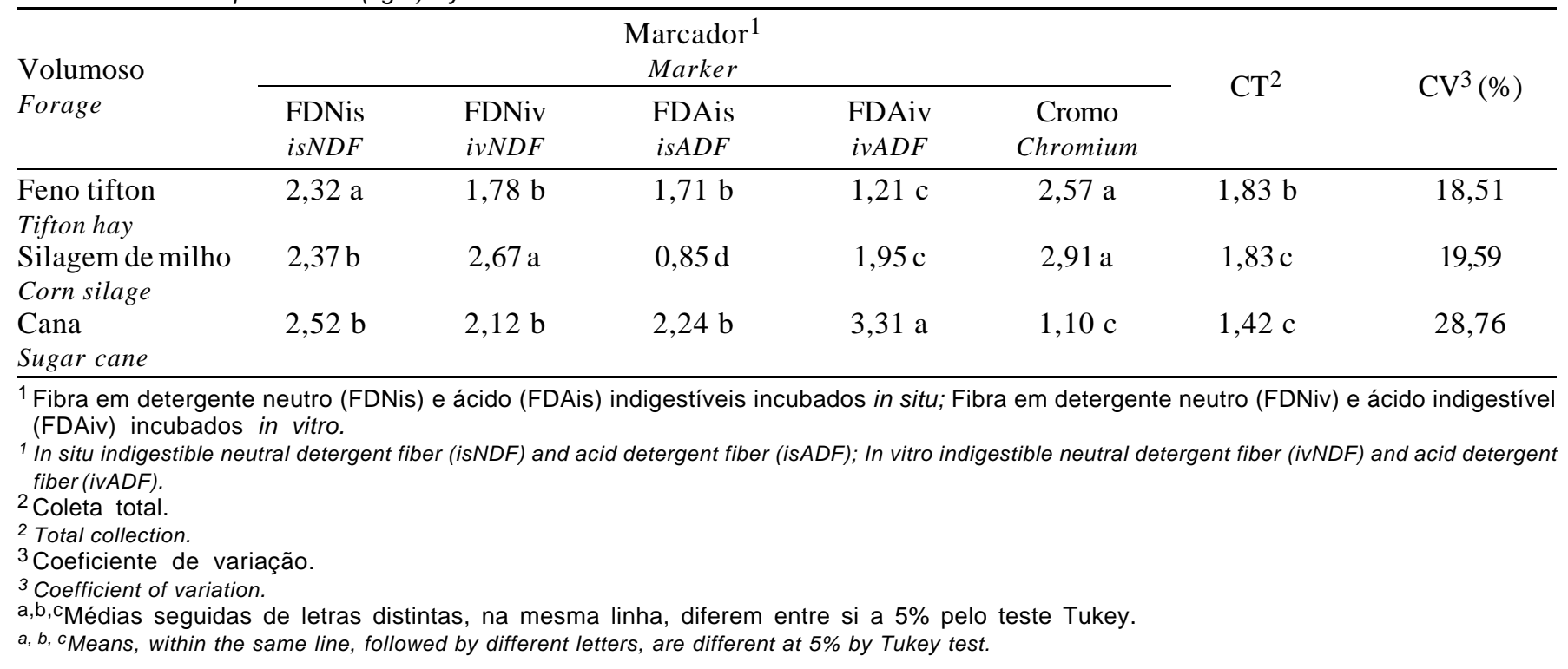

Outro aspecto que deve ser observado quando se trabalha com indicadores internos é a possibilidade de seleção das dietas pelos animais. Uma vez que o resíduo que sobra no cocho é, normalmente, rico em material indigestível, é importante que se realize uma correção nos dados de consumo do indicador em relação à fibra indigestível presente nas sobras.

No presente trabalho, apesar da tentativa de se controlar o consumo para evitar a presença de sobras no cocho e, com isso, a possibilidade de seleção, as dietas com cana-de-açúcar apresentaram baixo consumo, não sendo possível a ausência de sobras. No entanto, as amostras de sobras para este volumoso não foram incubadas, não sendo possível efetuar a correção necessária. Conseqüentemente, para grande parte dos parâmetros avaliados, as estimativas obtidas para cana-de-açúcar apresentaram maior coeficiente de variação, evidenciando a importância da correção no consumo da fibra indigestível quando há ocorrência de sobras.

Nas Figuras 1, 2 e 3, pode-se visualizar a distribuição da produção fecal estimada a partir dos marcadores em relação à coleta total.

Pela dispersão dos dados, diferenciada de acordo com cada gráfico, verifica-se novamente que os marcadores não apresentam a mesma relação com a CT nos diferentes volumosos.

A avaliação de recuperação dos marcadores internos e externo (Tabela 4) mostra que apenas o FDNiv e FDAis para o feno de Tifton, o FDAiv para a

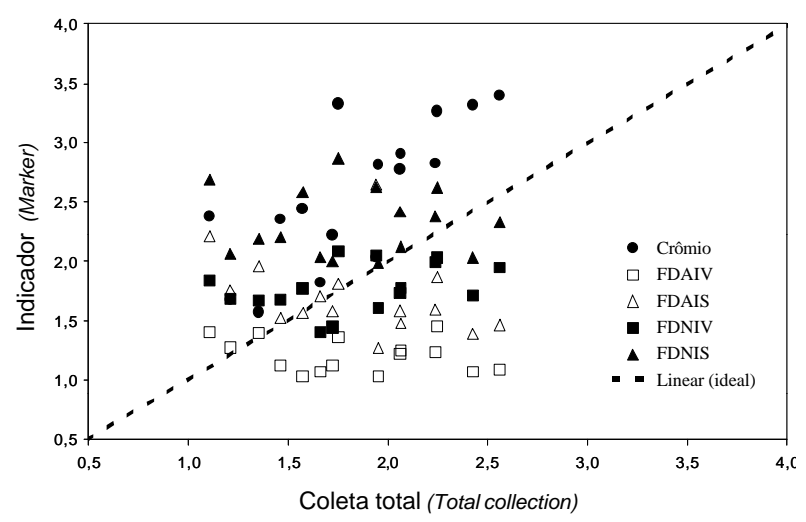

Figura 1 - Produção fecal estimada e coleta total de animais alimentados com feno de tifton.

Figure 1 - Estimative fecal production and total collection of animals fed hay Tifton.

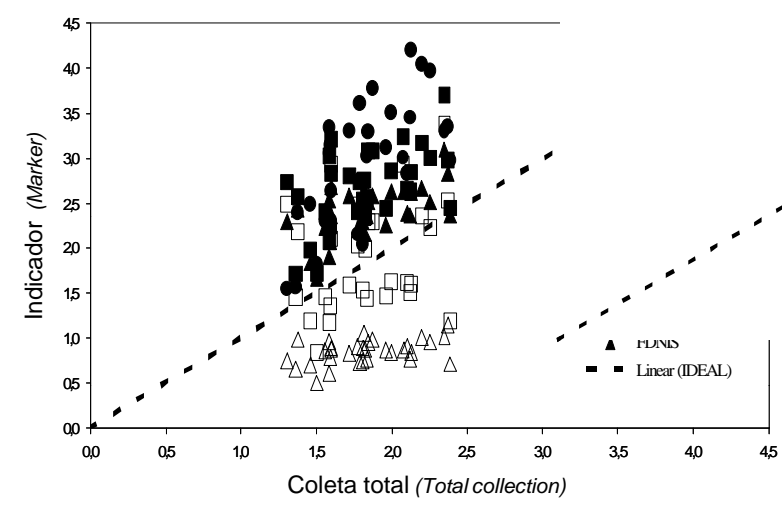

Figura 2 - Produção fecal estimada e coleta total de animais alimentados com silagem de milho.

Figure 2 - Estimative fecal production and total collection of animals fed corn silage. 


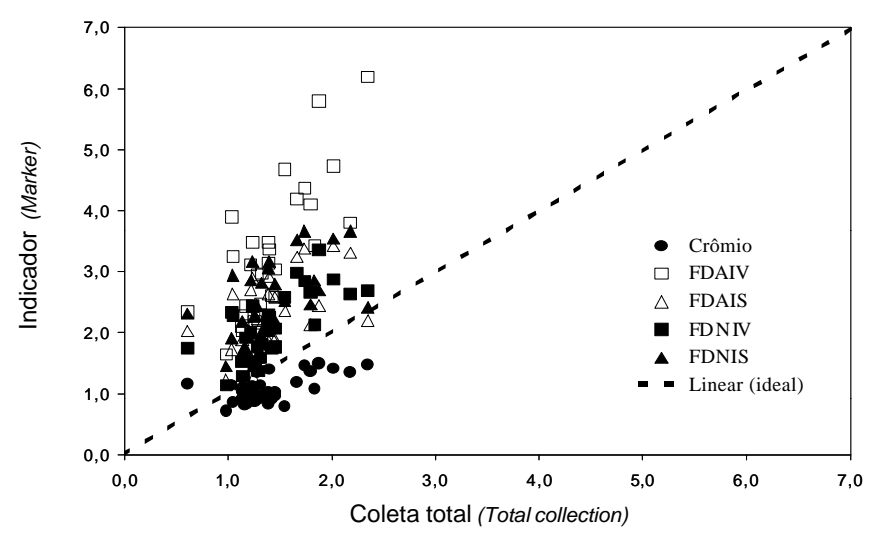

Figura 3 - Produção fecal estimada e coleta total de animais alimentados com cana-de-açúcar.

Figure 3 - Estimated fecal production and total collection of animals fed sugar cane.

silagem de milho e o cromo para a cana não diferiram de $100 \%$ de recuperação, estando de acordo com os resultados observados para a produção fecal estimada para os diferentes volumosos.

Os coeficientes de digestibilidade da matéria seca calculados a partir da produção fecal obtida pelo FDNiv e FDAis não diferiram da CT para os animais alimentados com feno de Tifton 85 (Tabela 5).

No caso da silagem de milho, apenas quando se utilizou o FDAiv para determinação da digestibilidade da MS não houve diferença quando comparada com a CT. Em relação à cana-de-açúcar, os coeficientes de digestibilidade diferiram entre si e quando comparados aos coeficientes determinados pela CT.
Em outros estudos (Krysl et al., 1988, Judkins et al., 1990; Huhtanen et al., 1994; Zeoula et al., 2000; Ítavo et al., 2002b), não houve acurácia da digestibilidade da matéria seca estimada pela obtenção da fibra indigestível in situ, resultando em subestimativa dos resultados. A subestimativa da digestibilidade da matéria seca sugere que a perda do marcador ocorre no processo digestivo do animal ou por meio das análises laboratoriais. Em outros estudos (Judkins et al., 1990; Nelson et al., 1990), o aumento do período de incubação dos componentes da fibra elevou a recuperação fecal do FDN e FDA indigestível, de modo que a recuperação fecal pode também ser função do tamanho de partícula do substrato fermentável (Lippke et al., 1986), sugerindo a necessidade de maior critério no preparo da amostra para incubação (Cochran et al., 1988).

Os resultados variados obtidos com FDN e FDA indigestível parecem ser decorrentes da perda de partículas de alimento e de amostras fecais através de sacos de náilon durante a incubação ruminal. Preparação de amostras para incubação no saco de náilon é provavelmente mais importante que para incubação in vitro. Para evitar perda de partículas através dos sacos, amostra de fezes e duodeno não devem ser moídas; concentrado e forragem devem ser picados ou moídos grosseiramente.

Tamminga et al. (1990) concluíram que a utilização de FDN indigestível determinada pela incubação de saco de náilon no rúmen pode não ser apropriada, podendo a FDA indigestível ser utilizada com maior

Tabela 4 - Recuperação percentual dos marcadores internos e externo nas fezes (\%) de diferentes volumosos Table 4 - Percentual recovery of internal and external markers (\%) in feces of different forages

\begin{tabular}{|c|c|c|c|c|c|c|}
\hline \multirow{2}{*}{$\begin{array}{l}\text { Volumoso } \\
\text { Forage }\end{array}$} & \multicolumn{5}{|c|}{$\begin{array}{c}\text { Marcador }^{1} \\
\text { Marker }\end{array}$} & \multirow{2}{*}{$\mathrm{CV}^{2}(\%)$} \\
\hline & $\begin{array}{l}\text { FDNis } \\
\text { is NDF }\end{array}$ & $\begin{array}{l}\text { FDNiv } \\
i v N D F\end{array}$ & $\begin{array}{l}\text { FDAis } \\
\text { isADF }\end{array}$ & $\begin{array}{l}\text { FDAiv } \\
i v A D F\end{array}$ & $\begin{array}{c}\text { Cromo } \\
\text { Chromium }\end{array}$ & \\
\hline $\begin{array}{l}\text { Feno tifton } \\
\text { Tifton hay }\end{array}$ & $127,08^{*}$ & 101,78 & 94,08 & $72,53 *$ & $142,73^{*}$ & 22,28 \\
\hline $\begin{array}{l}\text { Silagem de milho } \\
\text { Corn silage }\end{array}$ & $131,39^{*}$ & $148,12 *$ & $47,26^{*}$ & 108,38 & $159,18^{*}$ & 18,89 \\
\hline $\begin{array}{l}{ }^{1} \text { Fibra em detergen } \\
\text { indigestível (FDAiv } \\
{ }^{1} \text { In situ indigestible ne } \\
\text { fiber (ivADF). } \\
{ }^{2} \text { Coeficiente de var } \\
{ }^{2} \text { Coefficient of variatic } \\
{ }^{*} \text { Valores diferentes } \\
{ }^{*} \text { Different of } 100 \text { at } 5\end{array}$ & $\begin{array}{l}\text { leutro (FL } \\
\text { cubados } \\
\text { ldetergent } \\
\text { ào. } \\
100 \text { a } 5 \%\end{array}$ & $\begin{array}{l}\text { cido (FDA } \\
\text { F) and acid } \\
\text { e Tukey. }\end{array}$ & $\begin{array}{l}\text { estíveis } \\
\text { fiber (isA }\end{array}$ & $\begin{array}{l}\text { in situ; } \\
\text { indigestib }\end{array}$ & tetergente $r$ & $\begin{array}{l}\text { (FDNiv) e ácido } \\
\text { and acid detergent }\end{array}$ \\
\hline
\end{tabular}


Tabela 5 - Coeficiente de digestibilidade total da matéria seca (\%) para os diferentes volumosos Table 5 - Total digestibility coefficients of dry matter of different forages

\begin{tabular}{|c|c|c|c|c|c|c|c|}
\hline \multirow{2}{*}{$\begin{array}{l}\text { Volumoso } \\
\text { Forage }\end{array}$} & \multicolumn{5}{|c|}{$\begin{array}{c}\text { Marcador }^{1} \\
\text { Marker }\end{array}$} & \multirow{2}{*}{$\mathrm{CT}^{2}$} & \multirow{2}{*}{$\mathrm{CV}^{3}(\%)$} \\
\hline & $\begin{array}{l}\text { FDNis } \\
\text { is } N D F\end{array}$ & $\begin{array}{l}\text { FDNiv } \\
i v N D F\end{array}$ & $\begin{array}{l}\text { FDAis } \\
\text { is } A D F\end{array}$ & $\begin{array}{l}\text { FDAiv } \\
i v A D F\end{array}$ & $\begin{array}{c}\text { Cromo } \\
\text { Chromium }\end{array}$ & & \\
\hline $\begin{array}{l}\text { Feno tifton } \\
\text { Tifton hay }\end{array}$ & $51,18 \mathrm{c}$ & $62,72 \mathrm{~b}$ & $63,97 \mathrm{~b}$ & $73,77 \mathrm{a}$ & $45,99 \mathrm{c}$ & $61,41 \mathrm{~b}$ & 10,94 \\
\hline $\begin{array}{l}\text { Silagem de milho } \\
\text { Corn silage }\end{array}$ & $61,97 \mathrm{c}$ & $57,29 \mathrm{~d}$ & $86,36 \mathrm{a}$ & $69,18 b$ & $53,24 \mathrm{e}$ & $70,48 \mathrm{~b}$ & 8,14 \\
\hline $\begin{array}{l}\text { Cana } \\
\text { Sugar cane }\end{array}$ & $42,74 \mathrm{~d}$ & $51,79 \mathrm{c}$ & $49,43 \mathrm{c}$ & $30,50 \mathrm{e}$ & 74,45 a & $66,33 \mathrm{~b}$ & 13,04 \\
\hline
\end{tabular}

${ }^{1}$ Fibra em detergente neutro (FDNis) e ácido (FDAis) indigestíveis incubados in situ; Fibra em detergente neutro (FDNiv) e ácido indigestível (FDAiv) incubados in vitro.

1 In situ indigestible neutral detergent fiber (isNDF) and acid detergent fiber (isADF); In vitro indigestible neutral detergent fiber (ivNDF) and acid detergent fiber (ivADF).

2 Coleta total.

${ }^{2}$ Total collection.

3 Coeficiente de variação.

${ }^{3}$ Coefficient of variation.

a,b,c Médias seguidas de letras distintas, na mesma linha, diferem entre si a $5 \%$ segundo o teste Tukey.

$a, b, c$ Means, within the same line, followed by different letters, are different at $5 \%$ by Tukey test.

confiabilidade. No presente trabalho, no entanto, ambos os marcadores apresentaram variação dentro de cada volumoso.

Em decorrência da incapacidade para validar estimativas de fluxo digestivo com medidas de referência verdadeiras, os méritos e desvantagens dos métodos dos marcadores têm sido avaliados sobre a base de considerações teóricas (Ahvenjärvi et al., 2003). Considerando que um marcador, necessariamente, passa primeiro pelo duodeno antes de chegar às fezes, a completa recuperação no duodeno é provável em situação em que o marcador é recuperado completamente nas fezes (Titgemeyer, 1997).

A determinação do fluxo de matéria seca duodenal usando marcadores indigestíveis é baseada na relação entre sua concentração na digesta e a quantidade administrada. A falta de exatidão ao se determinar a concentração dos marcadores pode afetar a medida do fluxo da digesta (Siddons et al., 1985; Comb \& Satter, 1992).

Nas dietas contendo feno de tifton 85 e silagem de milho, o uso do óxido crômico superestimou o fluxo de matéria seca duodenal em comparação aos marcadores internos, independentemente do método de incubação (Tabela 6). Ainda para silagem de milho, os marcadores internos FDNiv e FDAiv resultaram em menores valores de fluxo que os estimados por FDNis e FDAis, indicando o efeito da metodologia para obtenção do marcador, o que comprova possível perda do material incubado para a metodologia in situ, resultando em superestimativa do fluxo de digesta duodenal em relação à metodologia in vitro.

O contrário ocorreu com a análise da cana-deaçúcar, observando-se que o fluxo duodenal de matéria seca estimado pelo marcador externo foi semelhante ao obtido pelos marcadores internos FDNis e FDAis, sendo inferiores ao valores de fluxo estimados com o FDNiv e FDAiv.

As diferenças entre cromo e FDN indigestível nas estimativas de fluxo duodenal parecem estar relacionadas a uma distribuição mais uniforme da FDN indigestível que do cromo na fase sólida, pois o cromo ligar-se-ia mais facilmente às partículas de menor tamanho. Considerando amostras de digesta obtidas com número maior de partículas pequenas em relação à digesta verdadeira e que maiores concentrações de cromo são encontradas em partículas menores, pode haver subestimativa do fluxo no duodeno (Huhtanen et al., 1994).

O uso de técnicas de reconstituição assume que o fluxo da digesta é semelhante a algum marcador fixo desde que a amostra coletada seja verdadeiramente representativa do que está fluindo no local (Faichney, 1975).

A não ser que um marcador seja uniformemente distribuído, amostras não representativas alteram a concentração do marcador em relação à sua concentração na digesta verdadeira, o que vai refletir como diferença nas estimativas de fluxo entre marcadores. 
Tabela 6 - Fluxo duodenal de matéria seca $(\mathrm{kg} / \mathrm{d})$ estimada com uso de marcadores Table 6 - Duodenal dry matter flow estimated by markers

\begin{tabular}{|c|c|c|c|c|c|c|}
\hline \multirow[b]{2}{*}{$\begin{array}{l}\text { Volumoso } \\
\text { Forage }\end{array}$} & \multicolumn{5}{|c|}{$\begin{array}{c}\text { Marcador }{ }^{1} \\
\text { Marker }\end{array}$} & \multirow[b]{2}{*}{$\mathrm{CV}^{2}(\%$} \\
\hline & $\begin{array}{l}\text { FDNis } \\
i s N D F\end{array}$ & $\begin{array}{l}\text { FDNiv } \\
i v N D F\end{array}$ & $\begin{array}{l}\text { FDAis } \\
\text { isADF }\end{array}$ & $\begin{array}{l}\text { FDAiv } \\
i v A D F\end{array}$ & $\begin{array}{l}\text { Cromo } \\
\text { Chromium }\end{array}$ & \\
\hline $\begin{array}{l}\text { Feno tifton } \\
\text { Tifton hay }\end{array}$ & $2,18 \mathrm{~b}$ & $2,34 \mathrm{~b}$ & $2,13 \mathrm{~b}$ & $2,26 \mathrm{~b}$ & $3,48 \mathrm{a}$ & 19,37 \\
\hline $\begin{array}{l}\text { Silagem de milho } \\
\text { Corn silage }\end{array}$ & $2,55 \mathrm{~b}$ & $1,99 \mathrm{c}$ & $2,71 b$ & $1,82 \mathrm{c}$ & $4,09 \mathrm{a}$ & 21,73 \\
\hline $\begin{array}{l}\text { Cana } \\
\text { Sugar cane }\end{array}$ & $2,25 \mathrm{~b}$ & $2,98 \mathrm{a}$ & $2,09 \mathrm{~b}$ & $3,10 \mathrm{a}$ & $1,91 \mathrm{~b}$ & 33,77 \\
\hline
\end{tabular}

${ }^{1}$ Fibra em detergente neutro (FDNis) e ácido (FDAis) indigestíveis incubados in situ; Fibra em detergente neutro (FDNiv) e ácido indigestível (FDAiv) incubados in vitro.

1 In situ indigestible neutral detergent fiber (isNDF) and acid detergent fiber (isADF); In vitro indigestible neutral detergent fiber (ivNDF) and acid detergent fiber (ivADF).

${ }^{2}$ Coeficiente de variação.

2 Coefficient of variation.

a,b,c Médias seguidas de letras distintas, na mesma linha, diferem entre si a $5 \%$ segundo o teste Tukey.

$a, b, c$ Means, within the same line, followed by different letters, are different at $5 \%$ by Tukey test.

Quando marcadores exibem variação significativa na distribuição entre fases da digesta pode ser uma indicação de extensão da amostragem não representativa. Todavia diferenças entre marcadores parecem também ocorrer em virtude de variações diurnas na concentração do marcador e de erros associados à determinação da sua concentração. Essas origens de variação podem ser minimizadas com freqüentes amostragens e métodos analíticos seguros para medir concentração dos marcadores (Gill et al., 1999).

Ahvenjärvi et al. (2003) relataram que, quando o fluxo da digesta é estimado usando o sistema de simples marcador, amostras não representativas afetam o fluxo da matéria seca, mas não têm efeito sobre a entidade química com a qual está intimamente associada.

A digestibilidade parcial do feno não apresentou diferença entre as estimativas obtidas pela determinação do fluxo da digesta duodenal baseada na concentração dos marcadores internos, entretanto, todos diferiram da digestibilidade parcial obtida pelo cromo (Tabela 7). Para silagem de milho e cana-de-açúcar, não houve diferença significativa entre FDNis e FDAis, assim como entre FDNiv e FDAiv. As digestibilidades parciais obtidas por intermédio do cromo não diferiram para cana-de-açúcar quando estimadas pelo FDNis e FDAis, diferindo quando se utilizou a metodologia in vitro.

A partição da digestão da fibra entre desaparecimento ruminal e pós-ruminal pode ser usada como indicativo para compreender se o comportamento de um marcador é adequado. Quando o fluxo de digesta total para o duodeno é subestimado, a digestão ruminal da fibra poderá ser superestimada e erroneamente indica que grande porção da fibra desaparece no rúmen. Em forragens de baixa qualidade, quase toda a digestão da fibra ocorre no rúmen (Köster et al., 1996). Com forragens de alta qualidade e alguns subprodutos com alto teor de fibra, uma porção da fibra potencialmente digestível pode sair do rúmen e ser digerida pós-rúmem, particularmente se a degradação de fibra foi inibida por adição de gordura à dieta (Pantoja et al., 1994).

No entanto, em ruminantes alimentados com forragens e subprodutos de boa qualidade, acima de $80 \%$ da digestão da fibra ocorrem no rúmen. Segundo Titgemeyer (1997), valores de digestão ruminal e no trato total dependem da dieta consumida e do nível de ingestão e, em dietas basicamente constituídas de forragem, a digestão ruminal é quase igual à digestão no trato total.

\section{Conclusões}

Os resultados do presente estudo permitem inferir que os componentes da fibra indigestível podem ser utilizados como marcadores. No entanto, pela grande variabilidade de resultados, observa-se, possivelmente, a existência de um marcador adequado para cada volumoso utilizado. Todavia, cuidados devem ser tomados na preparação da amostra, para se prevenir perdas diferenciais de partículas dos alimentos e das fezes, obtendo-se o melhor tempo e a melhor metodologia de incubação que irá refletir a fração indigestível. 
Tabela 7- Coeficientes de digestibilidade ruminal da matéria seca (\%) para os volumosos avaliados Table 7 - Ruminal digestibility coefficients of dry matter of different forages

\begin{tabular}{|c|c|c|c|c|c|c|}
\hline \multirow[b]{2}{*}{$\begin{array}{l}\text { Volumoso } \\
\text { Forage } \\
\end{array}$} & \multicolumn{5}{|c|}{$\begin{array}{c}\text { Marcador }^{1} \\
\text { Marker }\end{array}$} & \multirow[b]{2}{*}{$\mathrm{CV}^{2}(\%)$} \\
\hline & $\begin{array}{l}\text { FDNis } \\
\text { isNDF }\end{array}$ & $\begin{array}{l}\text { FDNiv } \\
i v N D F\end{array}$ & $\begin{array}{l}\text { FDAis } \\
\text { isADF }\end{array}$ & $\begin{array}{l}\text { FDAiv } \\
i v A D F\end{array}$ & $\begin{array}{c}\text { Cromo } \\
\text { Chromium }\end{array}$ & \\
\hline $\begin{array}{l}\text { Feno tifton } \\
\text { Tifton hay }\end{array}$ & $54,18 \mathrm{a}$ & $50,64 \mathrm{a}$ & $55,28 \mathrm{a}$ & $52,27 \mathrm{a}$ & $27,10 \mathrm{~b}$ & 19,73 \\
\hline $\begin{array}{l}\text { Silagem de milho } \\
\text { Corn silage }\end{array}$ & $58,82 \mathrm{~b}$ & $67,90 \mathrm{a}$ & $57,01 \mathrm{~b}$ & $70,58 \mathrm{a}$ & $34,18 \mathrm{c}$ & 14,92 \\
\hline
\end{tabular}

${ }^{1}$ Fibra em detergente neutro (FDNis) e ácido (FDAis) indigestíveis incubados in situ; Fibra em detergente neutro (FDNiv) e ácido indigestível (FDAiv) incubados in vitro.

1 In situ indigestible neutral detergent fiber (isNDF) and acid detergent fiber (isADF); In vitro indigestible neutral detergent fiber (ivNDF) and acid detergent fiber (ivADF).

${ }^{2}$ Coeficiente de variação.

2 Coefficient of variation.

a,b,c Médias seguidas de letras distintas, na mesma linha, diferem entre si a $5 \%$ segundo o teste Tukey.

$a, b, c$ Means, within the same line, followed by different letters, are different at $5 \%$ by Tukey test.

\section{Literatura Citada}

AGRICULTURAL AND FOOD RESEARCH COUNCIL AFRC. Technical committee on responses to nutrients: energy and protein requirements of ruminants. Wallingford: Commonwealth Agricultural Bureaux International, 1993. $159 \mathrm{p}$.

AHVENJÄRVI, S.; VANHATALO, A.; SHINGFIELD, K.J. et al. Determination of digesta flow entering the omasal canal of dairy cows using different marker systems. British Journal of Nutrition, v.90, n.1, p.41-52, 2003.

BERCHIELLI, T.T.; ANDRADE, P.; FURLAN, C.L. Avaliação de marcadores internos em ensaios de digestibilidade. Revista Brasileira de Zootecnia, v.29, n.3, p.830-833, 2000.

CARRILHO, E.N.V.M.; SILVEIRA, R.N.; SADER, A.P.O. et al. Decomposição de amostras via bomba de oxigênio para a determinação de $\mathrm{Cr}$ usado como marcador em estudos de digestibilidade em bovinos. In REUNIÃO ANUAL DA SOCIEDADE BRASILEIRA DE QUÍMICA, 26., 2003, Poços de Caldas. Anais... Poços de Caldas: Sociedade Brasileira de Química, 2003. QA-092.

COCHRAN, R.C.; VANZANT, E.S.; DelCURTO, T. Evaluation of internal markers isolated by alkaline hydrogen peroxide incubation and acid detergent lignin extraction. Journal of Animal Science, v.66, n.12, p.3245-3251, 1988.

COMBS, D.K.; SATTER, L.D. Determination of markers in digesta and feces by direct current plasma emission spectroscopy. Journal of Dairy Science, v.75, n.8, p.21762182, 1992.

FAICHNEY, G.J. The use of markers to partition digestion within the gastrointestinal tract. In: MacDONALD, I.W.; WARNER, A.A.I. (Eds.). Digestion and metabolism in the ruminant. Armidale: University of New England Publishing Unit, 1975. p.277-291.

FREITAS, D.; BERCHIELLI, T.T.; SILVEIRA, R.N. Produção fecal e fluxo duodenal de matéria seca e matéria orgânica estimados por meio de marcadores. Revista Brasileira de Zootecnia, v.31, n.3, p.1521-1530, 2002 (suplemento).
GILL, M.; ROBINSON, P.H.; KENNELLY, J.J. Diurnal patterns in rumen volume and composition of digesta flowing into the duodenum. Animal Science, v.69, n.1, p.237-249, 1999.

HUHTANEN, P.; KAUSTELL, K.; JAAKKOLA, S. The use of internal markers to predict total digestibility and duodenal flow of nutrients in cattle given six different diets. Animal Feed Science and Technology, v.48, n.1, p.211-227, 1994.

ÍTAVO, L.C.V.; VALADARES FILHO, S.C.; SILVA, F.F. et al. Comparação de marcadores e metodologia de coleta para estimativas de produção fecal e fluxo de digesta em bovinos. Revista Brasileira de Zootecnia, v.31, n.4, p.1833-1839, 2002a.

ÍTAVO, L.C.V.; VALADARES FILHO, S.C.; SILVA, F.F. et al. Consumo, degradabilidade ruminal e digestibilidade aparente de fenos de gramíneas do gênero Cynodon e rações concentradas utilizando marcadores internos. Revista Brasileira de Zootecnia, v.31, n.2, p.10241032, 2002b (Suplemento).

JUDKINS, M.B.; KRYSL, L.J.; BARTON, R.K. Estimating diet digestibility: a comparison of 11 techniques across six different diets fed to rams. Journal of Animal Science, v.68, n.5, p.1405-1415, 1990.

KÖSTER, H.H.; COCHRAN, R.C.; TITGEMEYER, E.C. et al. Effect of increasing degradation intake protein on intake and digestion of low-quality, tall-grass-prairie forage by beef cows. Journal of Animal Science, v.74, n.10, p. 2473-2481, 1996.

KRYSL, L.J.; GALYEAN, M.L.; ESTELL, R.E. et al. Estimating digestibility and faecal output in lambs using internal and markers. Journal of Agricultural Science, v.111, n.1, p.19-25, 1988.

LIPPKE, H.; ELLIS, W.C.; JACOBS, B.F. Recovery of indigestible fiber from feces of sheep and cattle on forage diets. Journal of Dairy Science, v.69, n.2, p.403-412, 1986.

MERCHEN, N.R. Digestion, absorption and excretion in ruminants. In: CHURCH, D.C. (Ed.) The ruminant animal digestive physiology and nutrition. Englewood Cliffs: Prentice-Hall, 1988. p.172-201.

NATIONAL RESEARCH COUNCIL - NRC. Nutrient requirements of dairy cattle. 7.ed. Washington, D.C.: National Academy of Science, 2001. 254p. 
NELSON, M.L.; MOTJOPE, L.; FINLEY, J.W. et al. Ash free indigestible acid detergent fiber as an internal marker to estimate digestibility wither grazing ruminants. Journal of Range Management, v.43, n.3, p.224-229, 1990.

OWENS, F.N.; HANSON, C.F. External and internal markers for appraising site and extent of digestion in ruminants. Journal of Dairy Science, v.75, n.9, p.2605-2617, 1992.

PANTOJA, J.; FIRKINS, J.L.; EASTRIDGE, M.L. et al. Effects of fat saturation and source of fiber on site of nutrient digestion and milk production by lactating dairy cows. Journal of Dairy Science, v.77, n.8, p.2314-2356, 1994,

RUIZ, R.; Van SOEST, P.J.; Van AMBURGH, M.E. et al. Use of chromium mordanted neutral detergent residue as a predictor of fecal output to estimate intake in grazing high producing Holstein cows. Animal Feed Science and Technology, v.89, n.1, p.155-164, 2001.

STATISTICAL ANALYSES SYSTEM - SAS. SAS users guide: Statistics, Version 8. Cary: 2003. 1028p.

SIDDONS, R.C.; PARADINE,J.; BEEVER, D.E. et al. Ytterbium acetate as a particulate-phase digesta - flow marker. British Journal of Nutrition, v.54, n.2, p.509-519, 1985.

TAMMINGA, S.; ROBINSON, P.H.; MEIJS, S. et al. Feed components as internal markers in digestion studies with dairy cows. Animal Feed Science and Technology, v.27, n.1, p.49-57, 1990.

TITGEMEYER, E.C. Design and interpretation of nutrient digestion studies. Journal of Animal Science, v.75, n.8, p.2235-2247, 1997.
VAN SOEST, P.J.; ROBERTSON, J.B.; LEWIS, B.A. Methods for dietary fiber, neutral detergent fiber, and nonstarch polysaccharides in relation to animal nutrition. Journal of Dairy Science, v.74, n.10, p.3583-3597, 1991.

WILLIAMS, C. H.; DAVID, D.J.; ILSMAA, O. The determination of chromic oxide in faeces samples by atomic absorption spectrophotometry. Journal of Agricultural Science, v.59, n.1, p.381-385, 1962.

ZEOULA, L.M.; KASSIES, M.P.; FREGADOLLI, F.L. et al. Uso de marcadores na determinação da digestibilidade parcial e total em bovinos. Acta Scientiarum, v.22, n.3, p.771-777, 2000.

ZEOULA, L.M.; PRADO, I.N.; DIAN, P.H.M. et al. Recuperação fecal de marcadores internos avaliados em ruminantes. Revista Brasileira de Zootecnia, v.31, n.4, p.1865-1874, 2002.
Recebido em: 02/04/03

Aceito em: 29/12/04 